\title{
Research Article \\ On the Structure of Brouwer Homeomorphisms Embeddable in a Flow
}

\section{Zbigniew Leśniak}

Institute of Mathematics, Pedagogical University of Cracow, Podchorążych 2, 30-084 Cracow, Poland

Correspondence should be addressed to Zbigniew Leśniak, zlesniak@up.krakow.pl

Received 10 May 2012; Accepted 25 July 2012

Academic Editor: Krzysztof Cieplinski

Copyright (C 2012 Zbigniew Leśniak. This is an open access article distributed under the Creative Commons Attribution License, which permits unrestricted use, distribution, and reproduction in any medium, provided the original work is properly cited.

We present two theorems describing the structure of the set of all regular points and the set of all irregular points for a Brouwer homeomorphism which is embeddable in a flow. The theorems are counterparts of structure theorems proved by Homma and Terasaka. To obtain our results, we use properties of the codivergence relation.

\section{Introduction}

Throughout the paper, $f$ will denote a Brouwer homeomorphism, that is, orientation preserving homeomorphism of the plane onto itself which has no fixed points.

For any sequence of subsets $\left(A_{n}\right)_{n \in \mathbb{Z}_{+}}$of the plane, we define limes superior $\limsup _{n \rightarrow \infty} A_{n}$ as the set of all points $p \in \mathbb{R}^{2}$ such that any neighbourhood of $p$ has common points with infinitely many elements of the sequence $\left(A_{n}\right)_{n \in \mathbb{N}}$. For any subset $B$ of the plane, we define the positive limit set $\omega_{f}(B)$ as the limes superior of the sequence of its iterates $\left(f^{n}(B)\right)_{n \in \mathbb{N}}$ and negative limit set $\alpha_{f}(B)$ as the limes superior of the sequence $\left(f^{-n}(B)\right)_{n \in \mathbb{N}}$. Under the assumption that $B$ is compact, Nakayama [1] proved that

$$
\begin{aligned}
& \omega_{f}(B)=\left\{q \in \mathbb{R}^{2}: \text { there exist sequences }\left(p_{j}\right)_{j \in \mathbb{N}} \text { and }\left(n_{j}\right)_{j \in \mathbb{N}}\right. \\
& \text { such that } \left.p_{j} \in B, n_{j} \in \mathbb{N}, n_{j} \longrightarrow+\infty, f^{n_{j}}\left(p_{j}\right) \longrightarrow q \text { as } j \longrightarrow+\infty\right\}, \\
& \alpha_{f}(B)=\left\{q \in \mathbb{R}^{2}: \text { there exist sequences }\left(p_{j}\right)_{j \in \mathbb{N}} \text { and }\left(n_{j}\right)_{j \in \mathbb{N}}\right. \\
& \text { such that } \left.p_{j} \in B, n_{j} \in \mathbb{N}, n_{j} \longrightarrow+\infty, f^{-n_{j}}\left(p_{j}\right) \longrightarrow q \text { as } j \longrightarrow+\infty\right\} .
\end{aligned}
$$


A point $p$ is called positively irregular if $\omega_{f}(B) \neq \emptyset$ for each Jordan domain $B$ containing $p$ in its interior, and negatively irregular if $\alpha_{f}(B) \neq \emptyset$ for each Jordan domain $B$ containing $p$ in its interior, where by a Jordan domain we mean the union of a Jordan curve $J$ and the Jordan region determined by $J$ (i.e., the bounded component of $\mathbb{R}^{2} \backslash J$ ). A point which is not positively irregular is said to be positively regular. Similarly, a point which is not negatively irregular is called negatively regular. A point which is positively or negatively irregular is called irregular, otherwise it is regular.

We say that a set $A \subset \mathbb{R}^{2}$ is invariant if $f(A)=A$. An invariant region $M$ is said to be parallelizable if there exists a homeomorphism $\varphi: M \rightarrow \mathbb{R}^{2}$ such that

$$
\left.f\right|_{M}=\varphi^{-1} \circ T \circ \varphi
$$

where $T$ is given by the formula $T(t, s)=(t+1, s)$. On account of the Brouwer Translation Theorem, for each $p \in \mathbb{R}^{2}$, there exists a parallelizable region $M$ containing $p$ (see [2]). This implies that a Brouwer homeomorphism looks locally like a translation. However, its global behaviour may be very complicated (cf. $[3,4])$.

For any $p \in \mathbb{R}^{2}$, one can construct an arc $K$ with endpoints $p$ and $f(p)$ such that $f(K) \cap K=\{f(p)\}$ (see [5]). Such an arc is called a translation arc. The Brouwer Lemma says that if $K$ is a translation arc, then $\bigcup_{n \in \mathbb{Z}} f^{n}(K)$ is a homeomorphic image of a straight line (see [2]). The set $\bigcup_{n \in \mathbb{Z}} f^{n}(K)$ is called a translation line. A translation line needs not be a topological line, where by a topological line we mean a closed set which is a homeomorphic image of a straight line.

Homma and Terasaka [6] proved two theorems describing the structure of a Brouwer homeomorphism. The theorems can be formulated in the following way.

Theorem 1.1 (see [6], First Structure Theorem). Let $f$ be a Brouwer homeomorphism. Then, the plane is divided into at most three kinds of pairwise disjoint sets: $\left\{O_{i}: i \in I\right\}$, where $I=\mathbb{N}$ or $I=\{1, \ldots, n\}$ for a positive integer $n,\left\{O_{i}^{\prime}: i \in \mathbb{N}\right\}$ and $F$. The sets $\left\{O_{i}: i \in I\right\}$ and $\left\{O_{i}^{\prime}: i \in \mathbb{N}\right\}$ are the components of the set of all regular points such that each $O_{i}$ is a parallelizable unbounded simply connected region, and each $O_{i}^{\prime}$ is a simply connected region satisfying the condition $O_{i}^{\prime} \cap f^{n}\left(O_{i}^{\prime}\right)=\emptyset$ for $n \in \mathbb{Z} \backslash\{0\}$. The set $F$ is invariant, closed, and consists of all irregular points.

Theorem 1.2 (see [6], Second Structure Theorem). Let $f$ be a Brouwer homeomorphism. Then, the plane is divided into at most three kinds of pairwise disjoint sets: $\left\{O_{i}: i \in I\right\}$, where $I=\mathbb{N}$ or $I=\{1, \ldots, n\}$ for a positive integer $n,\left\{O_{i}^{\prime}: i \in \mathbb{N}\right\}$ and $F$. The sets $\left\{O_{i}: i \in I\right\}$ and $\left\{O_{i}^{\prime}: i \in \mathbb{N}\right\}$ are the components of the set of all negatively regular points such that each $O_{i}$ is an invariant unbounded simply connected region and can be filled with a family of translation lines which are closed sets in $O_{i}$, and each $O_{i}^{\prime}$ is a simply connected region satisfying the condition $O_{i}^{\prime} \cap f^{n}\left(O_{i}^{\prime}\right)=\emptyset$ for $n \in \mathbb{Z} \backslash\{0\}$. The set $F$ is invariant, closed, and consists of all negatively irregular points.

The set $F$ occurring in the theorems above is the union of sets called singular lines and their cluster set. Homma and Terasaka [6] showed many properties describing mutual relationships among singular lines. Moreover, they proved that the set of all singular lines is at most countable. But the set $F$ occurring in the theorems above can also contain the cluster points of singular lines which do not belong to any singular line. Thus, to obtain the complete description of the set $F$, the study of the set of these cluster points is needed. In the case of an arbitrary Brouwer homeomorphism, the problem is still open. 
In this paper, we prove the counterparts of the structure theorems under the assumption that $f$ is embeddable in a flow. By a flow, we mean a group of homeomorphisms of the plane onto itself $\left\{f^{t}: t \in \mathbb{R}\right\}$ under the operation of composition which satisfies the following conditions:

(1) the function $\phi: \mathbb{R}^{2} \times \mathbb{R} \rightarrow \mathbb{R}^{2}, \phi(x, t)=f^{t}(x)$ is continuous,

(2) $f^{0}(x)=x$ for $x \in \mathbb{R}^{2}$,

(3) $f^{t}\left(f^{s}(x)\right)=f^{t+s}(x)$ for $x \in \mathbb{R}^{2}, t, s \in \mathbb{R}$.

We say that $f$ is embeddable in a flow if there exists a flow $\left\{f^{t}: t \in \mathbb{R}\right\}$ such that $f=f^{1}$.

\section{Codivergence Relation}

In this section, we characterize the sets of regular and irregular points of any Brouwer homeomorphism embeddable in a flow using the codivergence relation defined by Andrea [7].

For any Brouwer homeomorphism $f$, the codivergence relation is defined in the following way:

$p \sim q \quad$ if $p=q$ or $p$ and $q$ are endpoints of some arc $K$ for which $f^{n}(K) \longrightarrow \infty$ as $n \longrightarrow \pm \infty$.

By an $\operatorname{arc} K$ with endpoints $p$ and $q$, we mean the image of a homeomorphism $c:[0,1] \rightarrow$ $c([0,1])$ satisfying conditions $c(0)=p, c(1)=q$, where the topology on $c([0,1])$ is induced by the topology of $\mathbb{R}^{2}$.

It turns out that the relation defined above is an equivalence relation and under the assumption that $f$ is embeddable in a flow each equivalence class of the relation is an invariant simply connected set (see $[7,8])$.

Proposition 2.1. Let $f$ be a Brouwer homeomorphism which is embeddable in a flow $\left\{f^{t}: t \in \mathbb{R}\right\}$. Then, the set of all regular points is equal to the union of the interiors of all equivalence classes of the codivergence relation.

Proof. First we prove that every point $p$ belonging to the interior of an equivalence class $G_{0}$ is regular. By the definition of the interior, there exists a Jordan curve $J$ contained in $G_{0}$ such that the point $p$ belongs to the Jordan region $U$ whose boundary is equal to $J$. In the proof of the main theorem of [8], it has been showed that for every Jordan domain $B$ contained in an equivalence class which does not consist of just one orbit we have $f^{n}(B) \rightarrow \infty$ as $n \rightarrow \pm \infty$. Thus, $\omega_{f}(\mathrm{cl} U)=\emptyset$ and $\alpha_{f}(\mathrm{cl} U)=\emptyset$.

Conversely, if a point $p$ is regular, then there exists a Jordan region $U$ containing $p$ such that $f^{n}(\mathrm{cl} U) \rightarrow \infty$ as $n \rightarrow \pm \infty$. Since $U$ is arcwise connected, for each $q \in U \backslash\{p\}$ there exists an $\operatorname{arc} K$ with endpoints $p, q$ contained in $U$. Hence, $K$ satisfies the condition $f^{n}(K) \rightarrow \infty$ as $n \rightarrow \pm \infty$. Thus, each point of the Jordan region $U$ belongs to the same equivalence class as $p$. Consequently, $p$ belongs to the interior of this equivalence class.

From the proposition above, we obtain immediately the following. 
Corollary 2.2. Let $f$ be a Brouwer homeomorphism which is embeddable in a flow $\left\{f^{t}: t \in \mathbb{R}\right\}$. Then, the set of all irregular points is equal to the union of the boundaries of all equivalence classes of the codivergence relation.

\section{Structure of the Set of Regular Points}

In this section, we show an application of properties of the codivergence relation to describe the set of all regular points for a Brouwer homeomorphism $f$ which is embeddable in a flow.

Proposition 3.1. Let $f$ be a Brouwer homeomorphism which is embeddable in a flow $\left\{f^{t}: t \in \mathbb{R}\right\}$. Let $p$ be a regular point. Then, each point of the trajectory $C_{p}=\left\{f^{t}(p): t \in \mathbb{R}\right\}$ is a regular point.

Proof. Let $p$ be a regular point. Denote by $G_{0}$ the equivalence class which contains $p$. By Proposition 2.1, we have $p \in \operatorname{int} G_{0}$. Hence, the trajectory $C_{p}$ is contained in int $G_{0}$, since the interior of each equivalence class is invariant under any element of the flow $\left\{f^{t}: t \in \mathbb{R}\right\}$ (see [9]). Using Proposition 2.1 once again, we obtain that each element of the trajectory is a regular point.

In Theorem 1.1 describing the structure of any Brouwer homeomorphism, there are three types of sets: $O_{i}, O_{i}^{\prime}$, and $F$. Under the assumption that a Brouwer homeomorphism is embeddable in a flow, we only have two types of sets: $O_{i}$ and $F$. However, sets of type $O_{i}^{\prime}$ cannot occur.

Theorem 3.2. Let $f$ be a Brouwer homeomorphism which is embeddable in a flow $\left\{f^{t}: t \in \mathbb{R}\right\}$. Then, the plane is divided into at most two kinds of pairwise disjoint sets: $\left\{O_{i}: i \in I\right\}$, where $I=\mathbb{N}$ or $I=\{1, \ldots, n\}$ for a positive integer $n$, and $F$. The sets $\left\{O_{i}: i \in I\right\}$ are the components of the set of all regular points such that each $O_{i}$ is a parallelizable unbounded simply connected region. The set $F$ is closed and consists of all irregular points.

Proof. Suppose, on the contrary, that there exists a family of simply connected regions $\left\{O_{i}^{\prime}: i \in \mathbb{N}\right\}$ occurring in Theorem 1.1. Let us fix a point $p \in O_{i}^{\prime}$ for some $i \in \mathbb{N}$. Then, by Theorem 1.1, $p$ is a regular point and there exists a $j \in \mathbb{N}, j \neq i$ such that $f(p) \in O_{j}^{\prime}$.

By Proposition 3.1, each point of the trajectory $C_{p}$ is regular. In particular, all points belonging to the arc with endpoints $p$ and $f(p)$ contained in this trajectory are regular. On the other hand, the arc $K$ has to contain an irregular point, since $p$ and $f(p)$ belong to different components $O_{i}^{\prime}$ and $O_{j}^{\prime}$ of the sets of all regular points.

At the end of this section, let us note that the invariance of the set of all irregular points (and the set of all regular points) under each element of a flow $\left\{f^{t}: t \in \mathbb{R}\right\}$ such that $f=f^{1}$ can also be obtained from the relation $f=f^{-t} \circ f \circ f^{t}$ (see [10]).

\section{Structure of the Set of Irregular Points}

In this section, we proceed to study the structure of the set $F$ of all irregular points for a Brouwer homeomorphism $f$ which is embeddable in a flow $\left\{f^{t}: t \in \mathbb{R}\right\}$.

For any irregular point $p$, the set $P^{+}$is defined as the intersection of all $\omega_{f}(B)$ and the set $P^{-}$as the intersection of all $\alpha_{f}(B) \neq \emptyset$, where $B$ is a Jordan domain containing $p$ in its interior. An irregular point $p$ is strongly positively irregular if $P^{+} \neq \emptyset$, otherwise it is weakly positively irregular. Similarly, $p$ is strongly negatively irregular if $P^{-} \neq \emptyset$, otherwise it is weakly 
negatively irregular. We say that $p$ is strongly irregular if it is strongly positively irregular or strongly negatively irregular. Otherwise, an irregular point $p$ is said to be weakly irregular.

Nakayama [10] has proved that for any Brouwer homeomorphism the subset of $F$ consisting of all strongly irregular points has no interior points. In the case where $f$ is embeddable in a flow, the set $F$ is the union of a family of invariant topological lines, since the boundary of each equivalence class is the union of trajectories of the flow $\left\{f^{t}: t \in \mathbb{R}\right\}$ (see [9]). But some of these trajectories are not singular lines in the sense of Homma and Terasaka. The union of all singular lines is equal to the set of all strongly irregular points, and, moreover, the cluster points of singular lines which do not belong to any singular line are weakly irregular points (see [6]).

In the description of the set $F$, the notion of the first prolongational limit set can be used. For any point $p$, we define the first prolongational limit set of $p$ as $J(p)=J^{+}(p) \cup J^{-}(p)$, where

$$
\begin{aligned}
& J^{+}(p):=\left\{q \in \mathbb{R}^{2}: \text { there exist sequences }\left(p_{n}\right)_{n \in \mathbb{N}^{\prime}}\left(t_{n}\right)_{n \in \mathbb{N}}\right. \\
& \left.\quad \text { such that } p_{n} \longrightarrow p, t_{n} \longrightarrow+\infty, f^{t_{n}}\left(p_{n}\right) \longrightarrow q \text { as } n \longrightarrow+\infty\right\}, \\
& J^{-}(p):=\left\{q \in \mathbb{R}^{2}: \text { there exist sequences }\left(p_{n}\right)_{n \in \mathbb{N}^{\prime}}\left(t_{n}\right)_{n \in \mathbb{N}}\right. \\
& \text { such that } \left.p_{n} \longrightarrow p, t_{n} \longrightarrow-\infty, f^{t_{n}}\left(p_{n}\right) \longrightarrow q \text { as } n \longrightarrow+\infty\right\} .
\end{aligned}
$$

For an $H \subset \mathbb{R}^{2}$, we put

$$
J(H)=\bigcup_{p \in H} J(p)
$$

(see [11]). From the definition above, we obtain that

$$
p \in J^{+}(q) \Longleftrightarrow q \in J^{-}(p)
$$

for all $p, q \in \mathbb{R}^{2}$. Hence,

$$
J(p) \neq \emptyset \Longleftrightarrow p \in J\left(\mathbb{R}^{2}\right) .
$$

Proposition 4.1. Let $f$ be a Brouwer homeomorphism which is embeddable in a flow $\left\{f^{t}: t \in \mathbb{R}\right\}$. Let $p$ be a strongly irregular point. Then, $J(p) \neq \emptyset$.

Proof. Without loss of generality, we assume that $P^{+} \neq \emptyset$. We will show that $P^{+} \subset J^{+}(p)$. Let $q \in P^{+}$. For every positive integer $n$, we denote by $C_{n}$ the ball with centre $p$ and radius $1 / n$ and by $D_{n}$ the ball with centre $q$ and radius $1 / n$. Fix an $n \in \mathbb{N}$. Then, $q \in \omega_{f}\left(C_{n}\right)$. By the definition of $\omega_{f}\left(C_{n}\right)$, there exist sequences $\left(p_{j}\right)_{j \in \mathbb{N}}$ and $\left(m_{j}\right)_{j \in \mathbb{N}}$ such that $p_{j} \in C_{n}, m_{j} \in \mathbb{N}$, $m_{j} \rightarrow+\infty, f^{m_{j}}\left(p_{j}\right) \rightarrow q$ as $j \rightarrow+\infty$. Hence, there exists an $i \in \mathbb{N}$ such that $m_{i}>n$ and 
$f^{m_{i}}\left(p_{i}\right) \in D_{n}$. Put $q_{n}=p_{i}$ and $t_{n}=m_{i}$. Thus, we constructed sequences $\left(q_{n}\right)_{n \in \mathbb{N}}$ and $\left(t_{n}\right)_{n \in \mathbb{N}}$ such that

$$
q_{n} \in C_{n}, \quad t_{n}>n, \quad f^{t_{n}}\left(q_{n}\right) \in D_{n}
$$

for every $n \in \mathbb{N}$. Hence, $q_{n} \rightarrow p, t_{n} \rightarrow+\infty$ and $f^{t_{n}}\left(q_{n}\right) \rightarrow q$ as $n \rightarrow+\infty$. Consequently, $q \in J^{+}(p)$.

From the proposition above, we obtain the following.

Corollary 4.2. Let $f$ be a Brouwer homeomorphism which is embeddable in a flow $\left\{f^{t}: t \in \mathbb{R}\right\}$. Then, the set of all irregular points is equal to the closure of the first prolongational limit set of the plane.

Proof. By Proposition 4.1, if $p$ is a strongly irregular point, then $p \in J\left(\mathbb{R}^{2}\right)$. If $p$ is a weakly irregular point, then it belongs to the closure of the set of all strongly irregular points (see [6]). Consequently, $p \in \mathrm{cl} J\left(\mathbb{R}^{2}\right)$. The closure of the first prolongational limit set of the plane cannot contain any regular point, since for each $p$ belonging to the interior of an equivalence class we have $p \notin J\left(\mathbb{R}^{2}\right)$ (see [12]).

Using the main theorem of [13], we replace the regions $O_{i}$ occurring in Theorem 3.2 by larger parallelizable unbounded simply connected regions $U_{i}$ such that the union of all these regions $U_{i}$ contains the set of all weakly irregular points. A strongly irregular point can belong either to a region $U_{i}$ or to the set $F$. Moreover, for every singular line contained in the boundary of a region $U_{i}$, there can exist at most one singular line contained in the region (see [14]). Therefore, the counterpart of the Second Structure Theorem can be stated in the following way.

Theorem 4.3. Let $f$ be a Brouwer homeomorphism which is embeddable in a flow $\left\{f^{t}: t \in \mathbb{R}\right\}$. Then, the plane is divided into at most two kinds of pairwise disjoint sets: $\left\{U_{i}: i \in I\right\}$, where $I=\mathbb{N}$ or $I=\{1, \ldots, n\}$ for a positive integer $n$, and $F$. The sets $\left\{U_{i}: i \in I\right\}$ are parallelizable unbounded simply connected regions. The set $F$ is closed, contained in $J\left(\mathbb{R}^{2}\right)$, and is the union of at most countable family of trajectories of the flow. Each of these trajectories is contained in the boundary of an region $U_{i}$.

Using a decomposition described in the theorem above, we can obtain generalizations of results concerning Reeb homeomorphisms given by Béguin and Le Roux in [15].

\section{Final Remarks}

Let us consider the one-point compactification of a plane into the sphere $S^{2}$. Then, we can extend any Brouwer homeomorphism $f$ to a homeomorphism of the sphere by putting $f(\infty)=\infty$. Let us assume that $f$ is embeddable in a flow. Then, all trajectories are closed sets on the plane, since for all $p \in \mathbb{R}^{2}$ we have $f^{t}(p) \rightarrow \infty$ as $t \rightarrow \pm \infty$ (see [7]). Since the closure of each trajectory contains the stationary point $\infty$ of the flow, the phase portrait of the flow restricted to a Jordan region $U$ containing $\infty$ is divided into sectors (see [16], pages 161-174). 
The index of $\infty$ is equal to

$$
1+\frac{n_{e}-n_{h}}{2}
$$

where $n_{e}$ is the number of elliptic sectors and $n_{h}$ is the number of hyperbolic sectors (the expression gives an integer, since the difference of the number of elliptic sectors and the number of hyperbolic sectors is even). Applying the Lefschetz-Hopf Theorem to our case, we obtain that the index of the stationary point $\infty$ equals 2, since the Euler characteristic of the sphere equals 2 . In the case where $f$ is a translation, there are two elliptic sectors and two parabolic sectors. In the case where $f$ is a Reeb homeomorphism, there are three elliptic sectors, one hyperbolic sector and four parabolic sectors.

If a Jordan domain $B$ is contained in an elliptic sector of $U$, then $f^{n}(B)$ is contained in this sector for each $n \in \mathbb{Z}$. However, this property does not hold for parabolic and hyperbolic sectors. In the case where $f$ is a translation, for each Jordan region $U$ containing $\infty$ and each Jordan domain $B$ contained in one of the parabolic sectors, there exists an $n \in \mathbb{N}$ such that $f^{n}(B)$ is not contained in $U$. Thus even in case $f$ is a translation, the fixed point $\infty$ is not stable in the sense of the following definition: an invariant set $C$ is called Lyapunov stable if for any Jordan domain $U$ containing $C$ there is a Jordan domain $V$ containing $C$ such that $f^{n}(V) \subset U$ for all $n \in \mathbb{N}$ (see, e.g., [17]).

For a subset $D$ of the set of all homeomorphisms of a metric space $M$ equipped with the topology of uniform convergence on compact subsets, we say that $f \in D$ is structurally stable if there exists a neighborhood $U$ of $f$ in $D$ such that each $g \in U$ is topologically conjugate to $f$. If $M=\mathbb{R}^{2}$ and $D$ is the set of all Brouwer homeomorphisms, then there are no $f \in D$ which are structurally stable. Moreover, each of the topological conjugacy classes is dense in $D$ (see [18]).

Le Roux [19] gave a classification of the topological conjugacy classes of flows whose orbits are leaves of a given Reeb foliation of the plane. It could be interesting to study the structural stability of flows of Brouwer homeomorphisms. A flow $\left\{f^{t}: t \in \mathbb{R}\right\}$ is said to be structurally stable if for any flow $\left\{g^{t}: t \in \mathbb{R}\right\}$ in a neighbourhood of $\left\{f^{t}: t \in \mathbb{R}\right\}$ there is a homeomorphism $h: \mathbb{R}^{2} \rightarrow \mathbb{R}^{2}$ that sends the orbits of $\left\{f^{t}: t \in \mathbb{R}\right\}$ to the orbits of $\left\{g^{t}: t \in \mathbb{R}\right\}$ preserving the orientation of the orbits. This means that the phase portraits of the flows are homeomorphic.

\section{References}

[1] H. Nakayama, "Limit sets and square roots of homeomorphisms," Hiroshima Mathematical Journal, vol. 26, no. 2, pp. 405-413, 1996.

[2] L. E. J. Brouwer, “Beweis des ebenen Translationssatzes," Mathematische Annalen, vol. 72, no. 1, pp. $37-54,1912$.

[3] M. Brown, E. E. Slaminka, and W. Transue, "An orientation preserving fixed point free homeomorphism of the plane which admits no closed invariant line," Topology and its Applications, vol. 29, no. 3, pp. 213-217, 1988.

[4] E. W. Daw, "A maximally pathological Brouwer homeomorphism," Transactions of the American Mathematical Society, vol. 343, no. 2, pp. 559-573, 1994.

[5] P. Le Calvez and A. Sauzet, "Une démonstration dynamique du théorème de translation de Brouwer," Expositiones Mathematicae, vol. 14, no. 3, pp. 277-287, 1996.

[6] T. Homma and H. Terasaka, "On the structure of the plane translation of Brouwer," Osaka Journal of Mathematics, vol. 5, pp. 233-266, 1953. 
[7] S. A. Andrea, "On homoeomorphisms of the plane which have no fixed points," Abhandlungen aus dem Mathematischen Seminar der Universität Hamburg, vol. 30, pp. 61-74, 1967.

[8] Z. Leśniak, "On an equivalence relation for free mappings embeddable in a flow," International Journal of Bifurcation and Chaos in Applied Sciences and Engineering, vol. 13, no. 7, pp. 1911-1915, 2003.

[9] Z. Leśniak, "On parallelizability of flows of free mappings," Aequationes Mathematicae, vol. 71, no. 3, pp. 280-287, 2006.

[10] H. Nakayama, "On dimensions of non-Hausdorff sets for plane homeomorphisms," Journal of the Mathematical Society of Japan, vol. 47, no. 4, pp. 789-793, 1995.

[11] N. P. Bhatia and G. P. Szegö, Stability Theory of Dynamical Systems, Springer, New York, NY, USA, 1970.

[12] Z. Leśniak, "On maximal parallelizable regions of flows of the plane," International Journal of Pure and Applied Mathematics, vol. 30, no. 2, pp. 151-156, 2006.

[13] Z. Leśniak, "On a decomposition of the plane for a flow of free mappings," Publicationes Mathematicae Debrecen, vol. 75, no. 1-2, pp. 191-202, 2009.

[14] Z. Leśniak, "On boundaries of parallelizable regions of flows of free mappings," Abstract and Applied Analysis, vol. 2007, Article ID 31693, 8 pages, 2007.

[15] F. Béguin and F. Le Roux, "Ensemble oscillant d'un homéomorphisme de Brouwer, homéomorphismes de Reeb," Bulletin de la Société Mathématique de France, vol. 131, no. 2, pp. 149-210, 2003.

[16] P. Hartman, Ordinary Differential Equations, vol. 38, Society for Industrial and Applied Mathematics (SIAM), Philadelphia, Pa, USA, 2002.

[17] S. N. Elaydi, Discrete Chaos, Chapman \& Hall/CRC, Boca Raton, Fla, USA, 2nd edition, 2008.

[18] F. Le Roux, "Il n'y a pas de classification borélienne des homéomorphismes de Brouwer," Ergodic Theory and Dynamical Systems, vol. 21, no. 1, pp. 233-247, 2001.

[19] F. Le Roux, "Classes de conjugaison des flots du plan topologiquement équivalents au flot de Reeb," Comptes Rendus de l’Académie des Sciences. Série I. Mathématique, vol. 328, no. 1, pp. 45-50, 1999. 


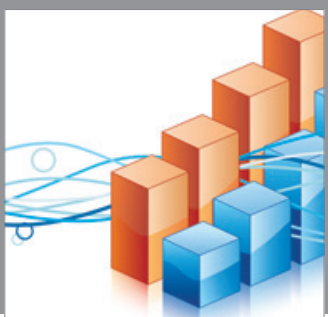

Advances in

Operations Research

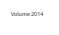

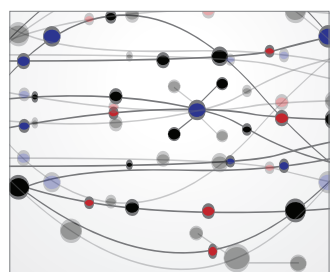

\section{The Scientific} World Journal
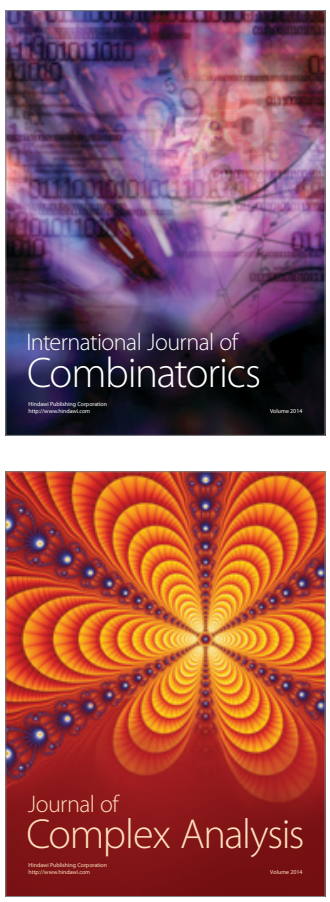

International Journal of

Mathematics and

Mathematical

Sciences
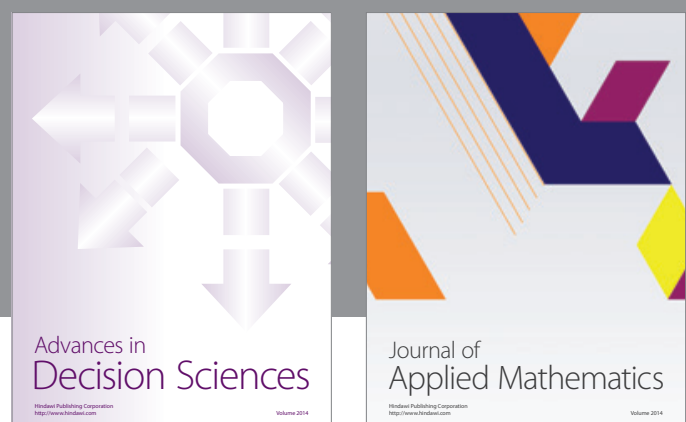

Journal of

Applied Mathematics
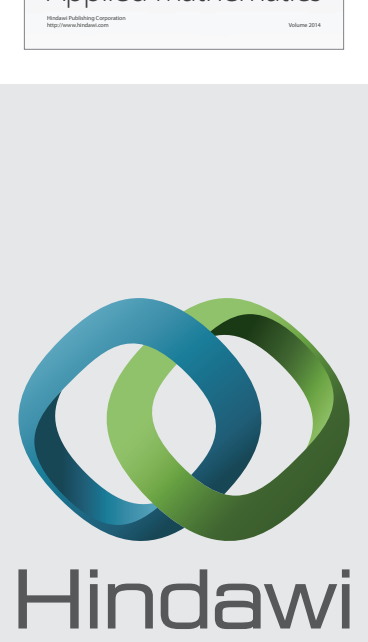

Submit your manuscripts at http://www.hindawi.com
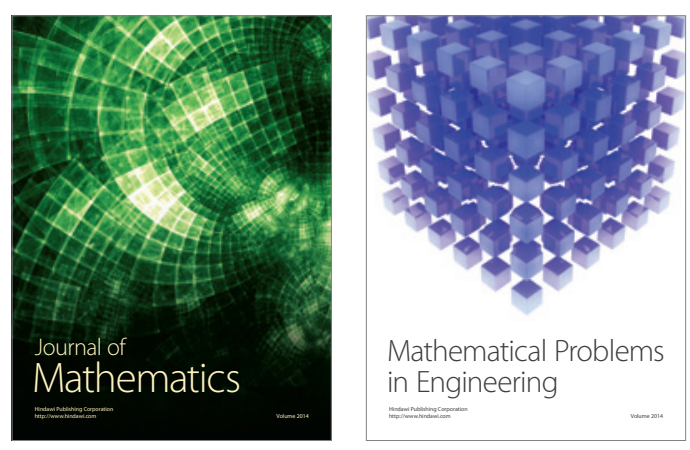

Mathematical Problems in Engineering
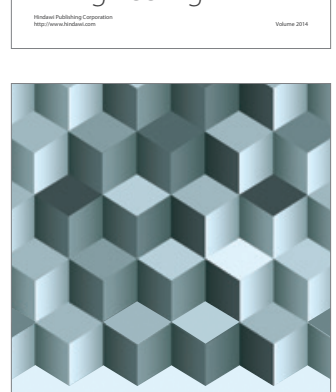

Journal of

Function Spaces
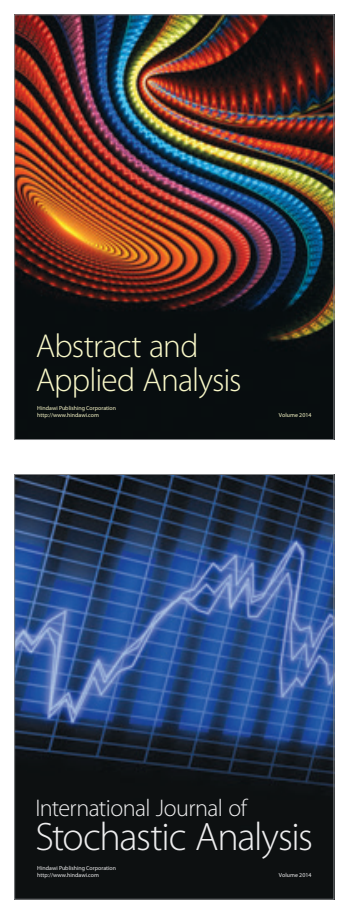

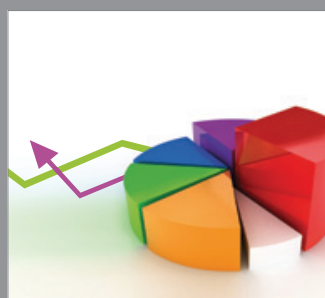

ournal of

Probability and Statistics

Promensencen
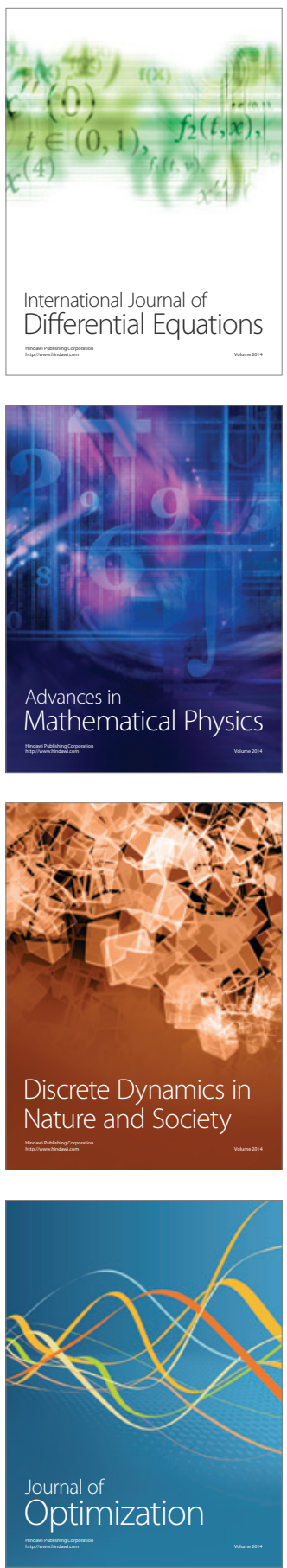